\title{
LOFAR and Radio-Loud AGN
}

\author{
Wendy L. Williams ${ }^{1,2}$ and Huub J. A. Röttgering ${ }^{1}$ on behalf of the \\ LOFAR collaboration \\ ${ }^{1}$ Leiden Observatory, Leiden University, P.O. Box 9513, 2300 RA Leiden, The Netherlands \\ ${ }^{2}$ Netherlands Institute for Radio Astronomy (ASTRON), PO Box 2, 7990AA Dwingeloo, \\ The Netherlands \\ emails: wwilliams@strw.leidenuniv.nl, rottgering@strw.leidenuniv.nl
}

\begin{abstract}
At very low frequencies, the new pan-European radio telescope, LOFAR, is opening the last unexplored window of the electromagnetic spectrum for astrophysical studies. Operating at frequencies from 15 to $240 \mathrm{MHz}$, its superb sensitivity, high angular resolution, large field of view and flexible spectroscopic capabilities represent a dramatic improvement over previous facilities at these wavelengths. LOFAR will carry out a broad range of fundamental astrophysical studies in a number of key science topics including the formation and evolution of clusters, galaxies and black holes. In this contribution we describe some of the capabilities of LOFAR and present some recent results from the ongoing imaging efforts. We also discuss the impact of LOFAR on our studies of radio-loud AGN. Our recent study of the evolution of radio-loud AGN as a function of host stellar mass shows a clear increase in the fraction of lower mass galaxies which host radio-loud AGN at $1<z<2$ while the fraction for higher mass galaxies remains the same. This shows that the upturn in the radio luminosity function is driven by increasing AGN activity among low mass galaxies at higher redshifts. New LOFAR observations will allow us to build statistically large samples at high redshifts to constrain this evolution for the different accretion modes of AGN.
\end{abstract}

Keywords. Galaxies: active, surveys, telescopes, Instrumentation: interferometers

\section{LOFAR Surveys and Recent Results}

The Low Frequency Radio Array (LOFAR) is a new low freqency radio telescope spanning several European countries. LOFAR has been described in detail elsewhere (see van Haarlem et al. 2013), but we provide here a few highlights: The array comprises several 'stations' each containing two groups of simple dipole antennas. The Low Band Array (LBA) operates at $10-80 \mathrm{MHz}$ and the High Band Array (HBA) at $110-240 \mathrm{MHz}$. There are 37 stations in the Netherlands, with baselines up to $\sim 80 \mathrm{~km}$, and a further eight stations in other European countries: five in Germany, and one each in the UK, Sweden, and France $\nmid$ which extends the array to $\sim 1200 \mathrm{~km}$. The electronically phased 'beams' enable multiple pointings to be observed simultaneously, making LOFAR extremely efficient for surveying large areas. They also make LOFAR highly flexible in terms of frequency choices.

The primary scientific drivers for LOFAR are encapsulated in the six Key Science Projects: (i) Epoch of Reionisation (EoR); (ii) Surveys; (iii) Transients; (iv) Cosmic Rays; (v) Cosmic Magnetism; and (vi) Solar Science and Space Weather. The Surveys project is most relevant here, and together with projects like Cosmic Magnetism, will provide images of nearby galaxies, AGN, clusters and large deep extragalactic fields. Three fundamental areas of astrophysics that have driven the design of the planned LOFAR

$\dagger$ up-to-date information on the current status of LOFAR is available at

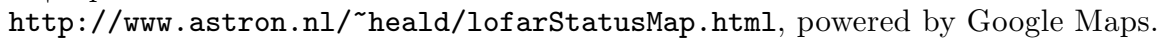


surveys are: (i) forming massive galaxies at the epoch of reionisation, (ii) magnetic fields and shocked hot gas associated with the first bound clusters of galaxies, and (iii) star formation processes in distant galaxies. To achieve the diverse and broad goals of the LOFAR surveys, a tiered approach will be used (for details see Röttgering et al. 2010): Tier-1 will be an all sky survey at 15, 30, 60 and $120 \mathrm{MHz}$; Tier-2 will be a medium deep survey over 1000 square degrees at 30,60, 120 and $200 \mathrm{MHz}$; and Tier-3 will cover about 100 square degrees down to an extreme depth of $6 \mu \mathrm{Jy} \mathrm{rms}$ at $150 \mathrm{MHz}$. The depth versus frequency of the proposed surveys is given in Fig. 1. Another important motivation of LOFAR is to provide the entire international astronomical community with unique surveys of the radio sky that have a long-lasting legacy value for a broad range of astrophysical research.

At present the measured noise in LOFAR images is dominated by calibration and imaging errors and some commissioning and technical research is still needed to obtain maps with the theoretical noise levels. However, the maps that are already being produced are the deepest ever at these low frequencies and high resolutions. Early-science observations are producing some excellent imaging results; we highlight here a few recent results. For nearby galaxies, (de Gasperin et al. 2012) have studied Virgo A (M87) in detail. The EoR group has produced images $15^{\prime \prime}$ resolution and noise levels of $100 \mu \mathrm{Jy} /$ beam across a wide band of 120-180 MHz (see Yatawatta et al. 2013, for some recent results) and their more recent work continues to push the noise levels down proportionally with increasing integration time. Extragalactic fields that have been imaged with LOFAR in the LBA include the Groth Strip and Boötes (see van Weeren et al. submitted) with images at $55-70 \mathrm{MHz}$ (noise 4-5 mJy, resolution 20" $)$, 40 - 50 MHz (6- $\left.7 \mathrm{mJy}, 30^{\prime \prime}\right)$, and $30-40$ $\mathrm{MHz}\left(11 \mathrm{mJy}, 40^{\prime \prime}\right)$. Current work includes that of directional dependent ionospheric calibration which results in noise levels of $\sim 100 \mu \mathrm{Jy}$ at $150 \mathrm{MHz}$ (in the Toothbrush cluster field, van Weeren in prep.) The first release of the Multi-frequency Sky Snapshot survey (MSSS; Heald et al. submitted) is expected soon. To date we have HBA observations to the Tier-1 depth of all the targeted famous extragalactic deep fields (including Boötes, Groth Strip, Lockman Hole, ELAIS-N1, COSMOS, GOODS-N, H-ATLAS, XMM-LSS, NEP) and several cluster fields (including the Toothbrush and Sausage clusters), and

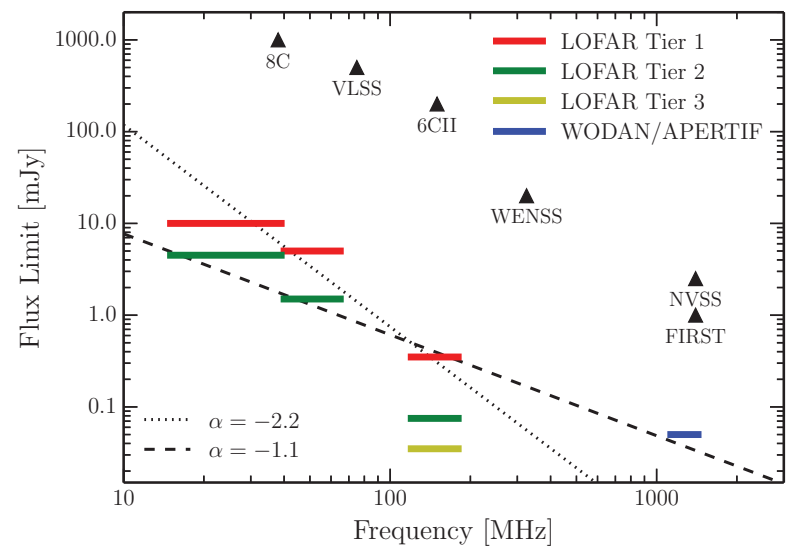

Figure 1. Flux limits $(5 \sigma)$ of the proposed LOFAR surveys compared to other existing radio surveys. The triangle represent existing surveys: NVSS, FIRST, WENSS, 6C, VLSS and 8C. The lines represent different power-laws $\left(S \sim \nu^{\alpha}\right.$ with $\alpha=-1.1$ and -2.2$)$ to illustrate how, depending on the spectral indices of the sources, the LOFAR surveys will compare to other current surveys. The proposed APERTIF/WODAN survey is also shown, which will use APERTIF on the WSRT. 
work is underway to produce the ionospheric-calibrated deep images. In Cycle 2 of LOFAR observations we initiated the Tier-1 HBA survey, by observing 42 pointings to the required Tier-1 depth. These first observations of this northern sky survey were chosen to lie within an area that is covered by Sloan Digital Sky Survey (SDSS) imaging and spectroscopy and is due to be covered by the Hobby-Eberly Telescope Dark Energy Experiment (HETDEX) in $2014-2017$.

\section{LOFAR and the evolution of radio loud AGN}

Radio galaxies have been shown to comprise two different populations: High and low excitation (Laing et al. 1994; Tadhunter et al. 1998), each which may have a separate and different feedback effect. The first mode, often referred to as 'cold-mode', 'radiativemode', or 'high-excitation', is characterised by strong optical emission lines, MIR emission and/or absorption associated with a dusty torus, along with possible X-ray emission. Here material is accreted on to the black hole through a radiatively efficient accretion disc. The second mode of AGN activity occurs with little radiated energy, but can still lead to the production of highly energetic radio jets. Here, the strong emission lines are absent and they lack other evidence for accretion disks. These 'radiatively inefficient', 'hot-mode', or 'low-excitation' AGN are thought to be fueled by radiatively inefficient advection-dominated accretion flows (ADAFs; e.g. Narayan \& Yi 1995).

The radio-loud AGN population is quite well-understood in the local universe within the context of these two accretion modes (see e.g. Best \& Heckman 2012; Janssen et al. 2012), however, this is not the case at higher redshifts. In particular, it remains unclear how the radio-loud AGN population evolves for different host stellar masses. The number density of powerful radio sources is two to three orders of magnitude greater at a redshift of two to three compared to the local Universe (e.g. Schmidt 1968; Rigby et al. 2011, and references therein). Locally, the fraction of galaxies which host a radio source, the radio-loud fraction, is a very steep function of host galaxy stellar mass (Best et al. 2005), increasing to $>30$ per cent at stellar masses above $5 \times 10^{11} \mathrm{M}_{\odot}$ for radio luminosities $>10^{23} \mathrm{~W} \mathrm{~Hz}^{-1}$. These two facts suggest that there must be an increase in the prevalence of radio activity among galaxies of lower mass.

Using the SDSS value-added spectroscopic sample of radio loud galaxies (Best \& Heckman 2012) and the VLA-COSMOS radio sample (Schinnerer et al. 2004, 2007) matched

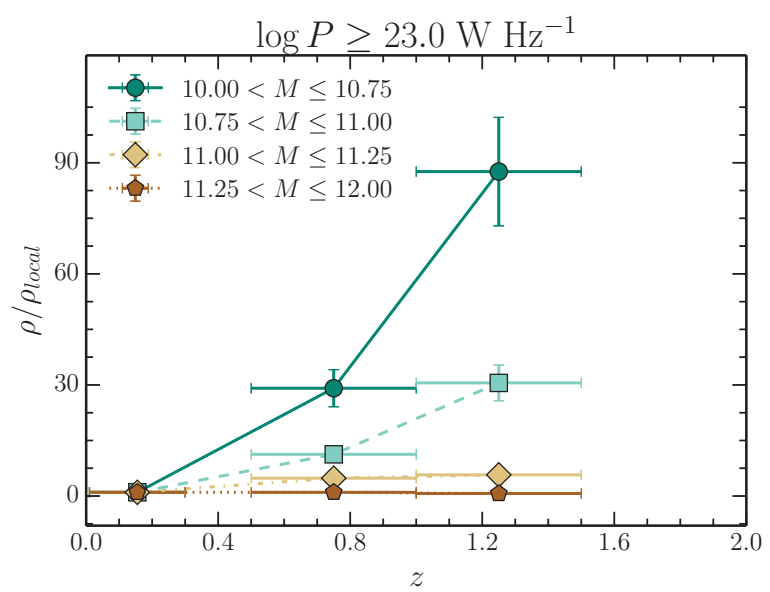

Figure 2. Relative space density of radio-loud sources with respect to the local density as a function of redshift for all sources with radio powers greater than the cutoff. 
to a $K_{s}$-selected catalog of the COSMOS/UltraVISTA field (Muzzin et al. 2013) to compile two samples of radio loud AGN going out to $z=2$. We have constructed radio luminosity functions binned by host stellar mass in three redshift ranges, $0.01 \leqslant z<0.3$, $0.5<z \leqslant 1.0$ and $1.0<z \leqslant 2.0$. Considering the relative comoving space density of radio-loud sources with respect to the local comoving space density, we find that at $1<z<2$ the space density of the least massive galaxies hosting radio-loud AGN is 90 times greater relative to the local space density, while that of higher mass galaxies hosting radio-loud AGN remains the same (see Fig. 2). The radio-loud fraction as a function of stellar mass is observed to be clearly increasing with redshift and becoming shallower with mass. Equivalently, the fraction of lower mass galaxies hosting radio sources increases faster with redshift than the fraction for high mass galaxies. This is likely driven by increasing cold-mode accretion driven by mergers at higher redshifts. This increase can thus explain the upturn in the Radio Luminosity Function (RLF) at high redshift which is important for understanding the impact of AGN feedback in galaxy evolution

\section{Prospects}

LOFAR has been producing excellent quality interferometric data for a number of years now. The commissioning period is over and Cycle 1 observations started in November 2013. LOFAR Surveys have already started imaging a handful of deep fields towards the surveys Tier-1 depth, and the target noise levels for the three tiers of the HBA Surveys are within reach. The combination of the LOFAR surveys data and other ancillary multiwavelength data available in many of the extra-galactic deep fields will make LOFAR a great tool for studies of AGN, distant clusters and galaxies.

\section{Acknowledgements}

LOFAR, the Low Frequency Array designed and constructed by ASTRON, has facilities in several countries, that are owned by various parties (each with their own funding sources), and that are collectively operated by the International LOFAR Telescope (ILT) foundation under a joint scientific policy.

\section{References}

Best, P. N., Kauffmann, G., Heckman, T. M., Brinchmann, J., et al. 2005, MNRAS, 362, 25

Best, P. N. \& Heckman, T. M. 2012 MNRAS, 421, 1569

de Gasperin, F., Orrú, E., Murgia, M., et al. 2012, A\&A, 547, A56

Hickox, R. C., Jones, C., Forman, W. R., et al. 2009, ApJ, 696, 891

Janssen, R. M. J., Röttgering, H. J. A., Best, P. N., \& Brinchmann, J., 2012, A\&A, 541, A62

Laing, R. A., Jenkins, C. R., Wall, J. V., et al. 1994, The Physics of Active Galaxies, 54, 201

Muzzin, A., Marchesini, D., Stefanon, M., et al. 2013, ApJS, 206, 8

Narayan, R. \& Yi, I. 1995, ApJ, 452, 710

Rigby, E. E., Best, P. N., Brookes, M. H., et al. 2011, MNRAS, 416, 1900

Röttgering, H. J. A. 2010, ISKAF2010 Science Meeting

Schinnerer, E., Carilli, C. L., Scoville, N. Z., et al. 2004, AJ, 128, 1974

Schinnerer, E., Smolčić, V., Carilli, C. L., et al. 2007, ApJS, 172, 46

Schmidt, M., 1968, ApJ, 151, 393

Tadhunter, C. N., Morganti, R., Robinson, A., et al. 1998, MNRAS, 298, 1035

van Haarlem, M. P., Wise, M. W., Gunst, A. W., et al. 2013, A\&A, 556, A2

Yatawatta, S., de Bruyn, A. G., Brentjens, M. A., et al. 2013, A\&AA, 550, A136 